\title{
Pneumococcal serotypes and respiratory failure: soil or seed?
}

\author{
Mark Woodhead
}

Affiliation: Dept of Respiratory Medicine, Manchester Royal Infirmary, Manchester, UK.

Correspondence: M. Woodhead, Dept of Respiratory Medicine, Manchester Royal Infirmary, Oxford Road, Manchester, M13 9WL, UK. E-mail: mark.woodheaddacmft.nhs.uk

0

@ERSpublications

Small differences in the capsule of causative bacteria may determine the exact pattern of clinical illness in pneumonia http://ow.ly/qlQbA

When considering the microbial aetiology of community-acquired pneumonia (CAP) we seldom think beyond the name (genus and species) of the causative organism. In a recent study of CAP aetiology in outpatients, Streptococcus pneumoniae was reported to be the most frequent pathogen [1]; unchanged from previous studies. The knowledge that $S$. pneumoniae is the commonest cause is sufficient to assist the choice of appropriate antibiotics; the most important management decision required for most patients.

However, there is a lot more to this bacterial species than the name might imply. In fact, there are now known to be $>90$ distinct pneumococcal serotypes, each characterised by a slightly different structure in the pneumococcal capsular polysaccharide. These small molecular differences are an important determinant of immune response; therefore, immunity is serotype specific. Studies in the pre-antibiotic era dealt in depth with serotype distributions, as at that time serotype specific serum was the only available therapy. With the advent of antibiotics, pneumococcal serotypes have received much less attention. Now with the development of pneumococcal vaccines, and the importance of matching vaccines to causative serotypes, this subject is having a renaissance. From this we are starting to understand that there is more to the different serotypes than just a difference in polysaccharide structure. Indeed, the serotype is increasingly being linked, not just with the immune response, but also with the clinical phenotype in individual patients.

In this issue of the European Respiratory Journal, BuRGOS et al. [2] describe an investigation into the risk factors for respiratory failure (defined as an oxygen saturation of $<90 \%$ on room air or an arterial oxygen tension/inspiratory oxygen fraction ratio $<250$ ) in a prospectively collected cohort of 1258 adults admitted to hospital with invasive (i.e. blood culture positive) pneumococcal pneumonia. Patients with respiratory failure (present in $48.9 \%$ of cases) were perhaps not surprisingly older, were more likely to have had chronic heart or lung disease, were more severely ill (as judged by the presence of septic shock and pneumonia severity index score), had more extensive radiographic change, were more likely to have received assisted ventilation and intensive care unit admission, and were more likely to have died. After multivariate analysis, age, chronic heart or lung disease, and pneumococcal serotypes 3,19A and 19F remained associated with respiratory failure, while type 1 remained associated with its absence.

This is the first study to find an association between specific serotypes and the presence or absence of respiratory failure. Previous studies have found associations between patterns of serotypes and other clinical phenotypes including nasopharyngeal carriage [3], invasive disease [4], septic shock [5], empyema and parapneumonic effusion [6], antibiotic resistance [7] and most importantly death [8]. These serotypic patterns are phenotypically specific so, for example, carriage serotypes tend not to be invasive and invasive serotypes tend not to cause death. The serotypes most associated with death in a recent meta-analysis were $3,6 \mathrm{~A}, 6 \mathrm{~B}, 9 \mathrm{~N}, 19 \mathrm{~A}, 19 \mathrm{~F}$ and $23 \mathrm{~F}$, whereas types $1,4,5,7 \mathrm{~F}$ and 8 had a reduced risk of death [8]. The three

Received: Aug 192013 | Accepted: Aug 202013

Conflict of interest: Disclosures can be found alongside the online version of this article at www.erj.ersjournals.com

Copyright @ERS 2014 
types found to be associated with respiratory failure in the current study are amongst those associated with increased mortality.

So why might the serotype be associated with the clinical phenotype? The most important feature identified to date is capsular thickness. The more encapsulated the pneumococcus the more likely it is to be an oropharyngeal commensal and the less likely it is to be invasive. However, if it does cause invasive disease then this is usually more severe. At first sight this might appear counter-intuitive. A thicker capsule allows the pneumococcus to evade the host defences and particularly phagocytosis, thus, favouring colonisation but at the same time this capsular thickness limits the ability to breach epithelial integrity and invade. Because of the protection from immune clearance favouring colonisation, if the organism does invade, more severe disease ensues. These disease patterns, at least until recently, seemed to be stable over time and across geographic locations.

The importance of the pneumococcal capsule as a target for immune defences and the association between a limited number of serotypes and invasive disease and death has facilitated the development of vaccines targeting a selected number of important serotypes, firstly with the polysaccharide and more recently the conjugate pneumococcal vaccines. However, use, particularly of the conjugate vaccines, is changing the ecology of the human-pneumococcal interface in ways that we are only just beginning to understand. Since the introduction of the 7-valent pneumococcal conjugate vaccine in the USA in 2000 there has been a sustained fall in frequency of isolation of the seven serotypes contained in the vaccine in both invasive and noninvasive isolates [9]. Initial concern that the seven serotypes would simply be replaced by nonvaccine types, while a reality for colonisation [10], has not materialised in clinical disease (perhaps due to differences in capsular structure) and has been translated into a dramatic reduction in pneumonia hospital admission and deaths, not just in vaccinated children, but also in the unvaccinated elderly [11, 12]. This appears to be a real step forward in reduction in pneumococcal disease.

There are two important caveats to the foregoing discussion. The first is that nearly all of the above is based on studies of invasive pneumococcal disease. The reasons are obvious; this represents a severe disease phenotype and is one that is easy to specifically identify. However, not all pneumococcal pneumonia is associated with invasive disease. Indeed, as much as $90 \%$ of adult pneumococcal pneumonia is not [13], and pneumonia associated with noninvasive serotypes has a higher mortality [13]. This may not matter if the pneumococcal serotypes that cause noninvasive and invasive pneumococcal pneumonia are the same. Difficulties in defining noninvasive pneumococcal disease have meant that until recently we have had little information about this. However, now with the availability of serotype specific urine antigen tests [14] we can begin to answer this question. The first such study showed that the invasive serotypes included in the 7 -valent conjugate vaccine accounted for only $20 \%$ of adult pneumococcal pneumonias, including serotypes covered by the new 13 -valent vaccine would have included $57 \%$ of cases. This needs to be investigated in other populations, but will need to be considered in future vaccine design.

Secondly, the clinical pneumococcal phenotype the result of the causative serotype or simply an artefact of host factors that have a propensity to facilitate pneumococcal disease with only certain serotypes? In the current study [2], attempts were made to control for host factors likely to be important, such as cerebrovascular disease, alcoholism and prior pneumococcal vaccination, which were no longer significant after multivariate analysis. While the aforementioned pneumococcal serotypes remained associated with respiratory failure, age and chronic respiratory and cardiac disease remained as predictors of respiratory failure. In another recent study of death in invasive pneumococcal disease, associations with specific pneumococcal serotypes were mitigated by host factors including age, alcoholism, liver or renal disease and the presence of solid tumours [15]. While methodological weaknesses in application of retrospective control for such factors may have led to spurious association with pneumococcal serotype, animal studies confirm that these are important in defining the clinical phenotype.

Vaccine use is changing the dynamics of the human-pneumococcal relationship before we fully understand the factors involved. Both host and bacterial factors will continue to be important in disease phenotype. Excitingly we are already seeing benefits from understanding this relationship, but continuing research and surveillance is going to be important in the success of future measures to control this disease.

\section{References}

Cilloniz C, Ewig S, Polverino E, et al. Community-acquired pneumonia in outpatients: aetiology and outcomes. Eur Respir J 2012; 40: 931-938.

2 Burgos J, Luján $\mathrm{M}$, Larrosa $\mathrm{MN}$, et al. Risk factors for respiratory failure in pneumococcal pneumonia: the importance of pneumococcal serotypes. Eur Respir J 2014; 43: 545-553.

3 Bogaert D, de Groot R, Hermans PWM. Streptococcus pneumoniae colonisation: the key to pneumococcal disease. Lancet Infect Dis 2004; 4: 144-154. 
4 Hausdorff WP, Bryant J, Paradiso PR, et al. Which pneumococcal serogroups cause the most invasive disease: implications for conjugate vaccine formulation and use, part I. Clin Infect Dis 2000; 30: 100-121.

5 Garcia-Vidal C, Ardanuy C, Tubau F, et al. Pneumococcal pneumonia presenting with septic shock: host- and pathogen-related factors and outcomes. Thorax 2010; 65: 77-81.

6 Bewick T, Sheppard C, Greenwood S, et al. Serotypes associated with the development of pneumococcal parapneumonic effusion in adults. Eur Respir J 2013; 42: 733-741.

7 Hackel M, Lascols C, Bouchillon S, et al. Serotype prevalence and antibiotic resistance in Streptococcus pneumonia clinical isolates among global populations. Vaccine 2013; 31: 4881-4887.

8 Weinberger DM, Harboe ZB, Sanders EAM, et al. Association of serotype with risk of death due to pneumococcal pneumonia: a meta-analysis. Clin Infect Dis 2010; 51: 692-699.

9 Richter SS, Heilmann KP, Dohrn CL, et al. Pneumococcal serotypes before and after introduction of conjugate vaccines, United States, 1999-2011. Emerg Infect Dis 2013; 19: 1074-1083.

10 Weinberger DM, Malley R, Lipsitch M. Serotype replacement in disease after pneumococcal vaccination. Lancet 2011; 378: 1962-1973.

11 Griffin MR, Zhu Y, Moore MR, et al. U.S. hospitalizations for pneumonia after a decade of pneumococcal vaccination. N Engl J Med 2013; 369: 155-163.

12 Simonsen L, Taylor RJ, Young-Xu Y, et al. Impact of pneumococcal conjugate vaccination of infants on pneumonia and influenza hospitalization and mortality in all age groups in the United States. MBio 2011; 2: e00309-10.

13 Bewick T, Sheppard C, Greenwood S, et al. Serotype prevalence in adults hospitalised with pneumococcal noninvasive community-acquired pneumonia. Thorax 2012; 67: 540-545.

14 Huijts SM, Pride MW, Vos JMI, et al. Diagnostic accuracy of a serotype-specific antigen test in communityacquired pneumonia. Eur Respir J 2013; 42: 1283-1290.

15 Naucler P, Jessica Darenberg J, Morfeldt E, et al. Contribution of host, bacterial factors and antibiotic treatment to mortality in adult patients with bacteraemic pneumococcal pneumonia. Thorax 2013; 68: 571-579. 